\title{
Modelado matemático de secado solar de orégano (Plectranthus amboinicus) con tecnologías directas e indirectas
}

\section{Mathematical model and solar drying of oregano (Plectranthus amboinicus) with direct and indirect technologies}

\author{
CASTILLO-TÉLLEZ, Beatriz†”， CASTILLO-TÉLLEZ-Margarita*”, MEJÍA-PÉREZ， Gerardo \\ Alberto""' y LÓPEZ VIDAÑA, Erick César"' \\ Centro Universitario del Norte, Universidad de Guadalajara, Colotlán, Jalisco, 46200, México. \\ "Facultad de Ingeniería, Universidad Autónoma de Campeche, Campeche, 24085, México. \\ "'"Centro de Investigación en Materiales Avanzados, S.C, Durango, Dgo., 34147, México. \\ "', Coordinación General de Extensión, Universidad de Guadalajara, Guadalajara, Jal., 44150, México.
}

ID $1^{\text {er }}$ Autor: Beatriz, Castillo-Téllez / ORC ID: 0000-0003-3747-6320, Researcher ID Thomson: S-2264-2018, CVU

CONACYT ID: 210564

ID $1^{\text {er }}$ Coautor: Margarita Castillo-Téllez / ORC ID: 0000-0001-9639-1736, Researcher IDThomson: S-2283-2018, CVU

CONACYT ID: 210428

ID $2^{\text {do }}$ Coautor: Erick César López Vidaña / /ORC ID: 0000-0002-9200-6578, CVU CONACYT-ID: 333477

ID $3^{\text {er }}$ Coautor: Gerardo Alberto, Mejía Pérez / ORC ID: 0000-0003-1701-1171

DOI: $10.35429 /$ JTIP.2020.10.4.27.36

Recibido: Enero 25, 2020; Aceptado: Junio 30, 2020

\begin{abstract}
Resumen
El orégano es una planta con muchas propiedades nutricionales y medicinales, además de tener otras aplicaciones en la industria química. En el presente trabajo se estudian las cinéticas de secado de hojas de orégano utilizando un secador tipo gabinete con y sin convención forzada, secado a cielo abierto y secado solar indirecto. Las muestras tuvieron un contenido de humedad inicial de 80 y final de $9 \%$. Los resultados indican que el secado solar indirecto provee un mejor control de las condiciones de operación y mayor protección contra los efectos de temperatura, produciendo una mejor calidad en el producto seco. El tiempo necesario para alcanzar el contenido de humedad de equilibrio en todos los experimentos varió entre 375 y $600 \mathrm{~min}$. Adicionalmente, los datos se ajustaron a diversos modelos matemáticos, resultando Weibull, Logarítmico y Page los que mejor representan el comportamiento del secado de las hojas de orégano. Los estadísticos utilizados obtenidos para el secador solar indirecto son $\mathrm{R}^{2}$ de $0.9969,0.9968$, y $0.9945, \mathrm{X}^{2}$ de $0.0363,0.0363$ y 0.0599 respectivamente.
\end{abstract}

Secado solar de orégano, Secado solar, Modelos de secado

\begin{abstract}
Oregano is a plant with many nutritional and medicinal properties; there are also other applications in the chemical industry. In this work, the drying kinetics of oregano leaves are studied using a cabinet-type dryer with and without a forced convention, open sun drying, and indirect solar drying. The samples had an initial moisture content of $80 \%$ and the final content of $9 \%$. The results indicate that indirect solar drying provides better control of operating conditions and greater protection against temperature effects, producing better quality in the dry product. The time required to reach the equilibrium moisture content in all experiments ranged from 375 to $600 \mathrm{~min}$. The data were also adjusted to various mathematical models, resulting In Weibull, Logarithmic, and Page, which best represent the drying behavior of oregano leaves. The statistics used for indirect solar dryer are $\mathrm{R}^{2}$ of $0.9969,0.9968$, and $0.9945, X^{2}$ of $0.0363,0.0363$ and 0.0599 respectively.
\end{abstract}

Solar drying of oregano, Solar drying, Drying models

Citación: CASTILLO-TÉLLEZ, Beatriz, CASTILLO-TÉLLEZ-Margarita, MEJÍA-PÉREZ, Gerardo Alberto y LÓPEZ VIDAÑA, Erick César. Modelado matemático de secado solar de orégano (Plectranthus amboinicus) con tecnologías directas e indirectas. Revista de Tecnologías en Procesos Industriales. 2020. 4-10:27.36.

\footnotetext{
* Correspondencia del Autor (Email: mcastill@uacam.mx)

$\uparrow$ Investigador contribuido como primer autor.
} 


\section{Introducción}

El orégano es una hierba cultivada con fines culinarios desde tiempos remotos, pero en la actualidad se utiliza en perfumes, medicamentos, bebidas alcohólicas, como conservador en alimentos, cosméticos, aceites automotrices y de aeronáutica y recientemente se ha encontrado que ayuda contra enfermedades como la hiperglucemia, hipertensión, obesidad (Mueller et al., 2008).

Ha encontrado como hábitat sitios secos y pedregosos donde sería imposible cosechar otros cultivos e incluso en lugares como el Norte de Jalisco, (donde se cosecha entre 800 y 1000 ton al año), Zacatecas, Coahuila y Chihuahua, el orégano contiene hasta un $30 \%$ más de aceite, incrementando su valor comercial (Vázquez Martínez, 2010).

El orégano en general se utiliza seco. La deshidratación del producto es una solución que impide el crecimiento de microorganismos, alarga su vida útil y puede prevenir ciertos cambios bioquímicos (Hossain et al., 2010). Comúnmente en México, tanto para alimentos como para medicamentos o extracción de aceites este proceso se realiza a cielo abierto, es decir, se arranca de raíz el arbusto y se deja en el sitio el tiempo necesario para su secado, para posteriormente sacudirlo y llevarlo a empresas que lo empaquetan y lo comercializan en el país o en el extranjero (Vázquez, 2016). Este proceso es por demás, aunque económico, poco eficiente e higiénico, ya que el producto queda expuesto a polvos, roedores, lluvia, o cualquier otra condición ambiental. etc.

El secador solar es un dispositivo que pueden ofrecer procesos de secado más rápidos con un secado más uniforme, higiénico y efectivo que los métodos convencionales, logrando obtener productos secos con mejor color y textura (Castillo Téllez et al., 2018). Una amplia variedad de tecnologías de secado solar han sido reportadas por muchos investigadores en el campo, que incluyen secadores solares directos, indirectos e híbridos (Chouicha et al., 2013; Fudholi et al., 2014; Méndez-Robles et al., 2018; Tlatelpa-Becerro et al., 2018).
El secado de los alimentos depende del calor y las características de transferencia de masa del producto que se seca. Un conocimiento de la distribución de la temperatura y la humedad en el producto es vital para el diseño de equipos y procesos, control de calidad, elección de almacenamiento adecuado y prácticas de su manejo. Los modelos matemáticos que describen los mecanismos de secado de los alimentos pueden proporcionar la información de temperatura y humedad requerida (Doymaz and Smail, 2011; Tunde-Akintunde, 2011; Ouaabou et al., 2018).

En este trabajo, presentan las cinéticas de secado solar de orégano en tecnologías directas e indirectas. Además, los resultados experimentales se han comparado con los modelos matemáticos de secado de capa delgada, lo cual nos permite predecir algunas variables en diferentes condiciones, así como aportar información para un diseño adecuado de secadores que permitan optimizar el proceso de secado solar de orégano, conservando sus propiedades organolépticas y nutritivas de una manera sustentable.

\section{Materiales y métodos}

Los experimentos se realizaron en la Universidad Autónoma de Campeche, Campeche, con coordenadas 19 $51^{\circ} 00^{\prime \prime} \mathrm{N}$ y $19^{\circ} 51^{\prime} 00^{\prime \prime} \mathrm{W}$. Este sitio tiene un clima tropical húmedo y seco, con $74 \%$ de humedad relativa promedio anual, temperatura promedio máxima de $32.7^{\circ} \mathrm{C}$ y un valor promedio anual global de radiación solar de $5.8 \mathrm{kWh} / \mathrm{m}^{2} /$ día. El período de prueba fue del 1 de julio al 30 de septiembre de 2019.

\section{Materia prima}

Se seleccionó el orégano (Plectranthus amboinicus (Lour.)), cultivado en el municipio de Macuspana, Tabasco. Las ramas se cortaron y las hojas se separaron para obtener un grupo homogéneo basado en su madurez, color y frescura. Las hojas se lavaron y pesaron, y se midió su anchura, longitud y grosor. No hubo diferencias significativas en cuanto al tamaño y el peso de las diferentes plantas seleccionadas para la prueba. Se utilizó una muestra de $20 \mathrm{~g}$ de hoja de orégano para cada prueba. 


\section{Métodos experimentales}

Secado Solar Directo (DSD)

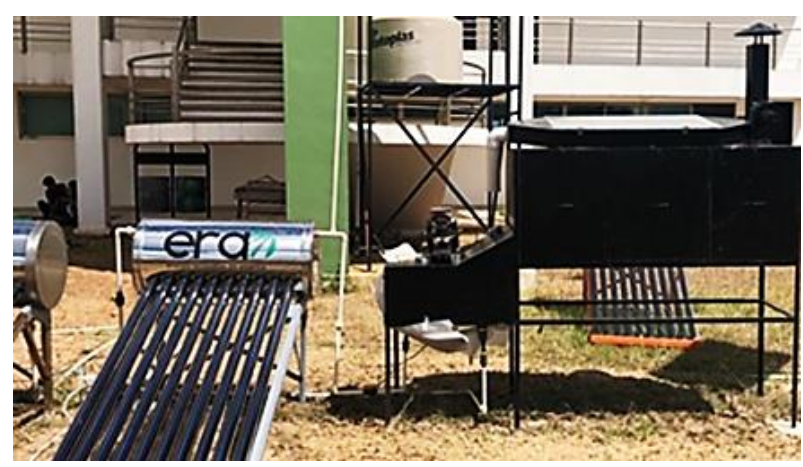

Figura 1 Secador solar indirecto. a) Cámara de secado, b) Chimenea y c) Colector de aire

Se utilizó un secador solar directo tipo gabinete con material plástico transparente y una superficie de tratamiento de $0.5 \mathrm{~m}^{2}$. La cámara de secado contiene una superficie que absorbe la radiación solar, en la que se coloca el producto a secar. Las partes laterales, inferiores y posteriores están perforadas para permitir la circulación y extracción de aire caliente y húmedo. El DSD se puede operar con circulación de aire natural o convección forzada utilizando un ventilador con una velocidad de aire máxima de $2 \mathrm{~m} / \mathrm{s}$.

Se midieron la temperatura interior, la cantidad de pérdida de peso en las muestras, la irradiancia solar, la humedad relativa y la temperatura del aire. La Figura 1 a) muestra los secadores solares directos que se utilizaron en este estudio.

Las pruebas se realizaron simultáneamente utilizando dos DSD. En uno de ellos se cubrió con una mala con filtración solar del 60\%. En ambos casos, los secadores solares, se probaron en condiciones ambientales similares.

\section{Secado a cielo abierto}

En este proceso, las hojas de orégano se colocan sobre una pieza de plástico adecuada como se muestra en la Figura 2. b) sin sobreponerlas y se dejan hasta el atardecer. Si las hojas no están completamente secas, se recolectan y almacenan en el interior. Este proceso se repite día tras día hasta que se completa el proceso de secado.

\section{Secado solar indirecto (ISD)}

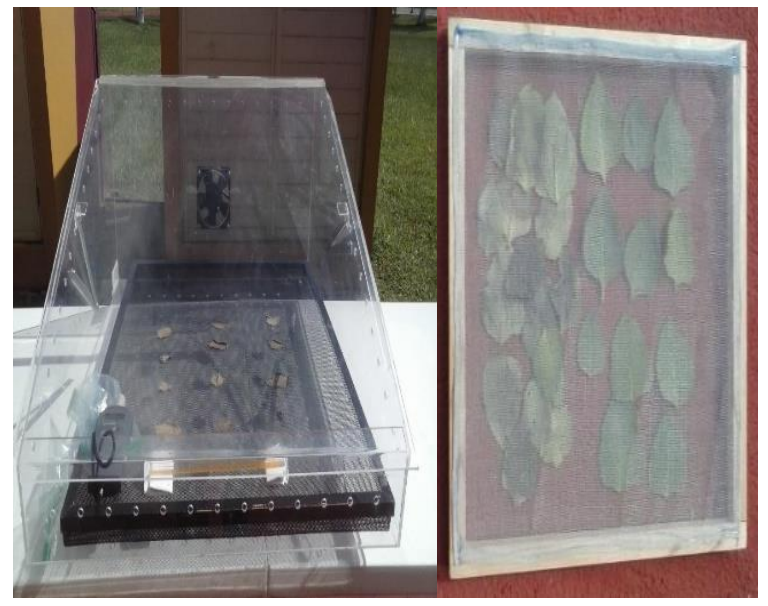

Figura 2 a) Secador solar directo con convección forzada y b) Secado a cielo abierto

El secador solar indirecto (ISD) consta de a) cámara de secado, b) chimenea y c) colector de aire. La cámara de secado es un túnel rectangular horizontal con una longitud de 0.47 $\mathrm{m}$ y una sección transversal de $0.16 \mathrm{~m}^{2}$. Está construido de madera con aislamiento térmico y se divide en tres niveles con tres bandejas para contener el producto a secar Figura 3.

\section{Modelado de las cinéticas de secado}

Para analizar el comportamiento de secado de diferentes productos agrícolas, se han propuesto previamente modelos matemáticos para describir su cinética de secado por convección. El proceso de secado en la mayoría de estos modelos se considera bajo condiciones isotérmicas; por lo tanto, se consideró un mecanismo de transferencia de masa difusional. Los modelos más ampliamente utilizados se enumeran en la Tabla 1. Se puede observar que la relación de humedad (MR) se considera como una función del tiempo de secado, que se puede calcular como:

$M R=\frac{M-M_{e}}{M_{0}-M_{e}}$

Donde $\mathrm{M}$ es el contenido de humedad (el cociente entre el peso del contenido en agua en la muestra fresca y el peso de la muestra después de la sequedad), $\mathrm{M}_{\mathrm{e}}$ es la humedad de equilibrio (bajo temperatura controlada específica $\mathrm{y}$ humedad del aire durante un largo periodo de tiempo), y $\mathrm{M}_{0}$ es la humedad inicial. 


\begin{tabular}{|c|c|c|}
\hline Modelo & Ecuación & Referencia \\
\hline Newton & $M R=\exp (-k t)$ & $\begin{array}{l}\text { (Liu and Bakker- } \\
\text { Arkema, 1997) }\end{array}$ \\
\hline Page & $M R=\exp \left(-k t^{n}\right)$ & (Page, 1949) \\
\hline $\begin{array}{l}\text { Modified } \\
\text { Page }\end{array}$ & $M R=\exp \left(-(k t)^{n}\right)$ & $\begin{array}{l}\text { (White, Ross and } \\
\text { Poneleit, 1981) }\end{array}$ \\
\hline $\begin{array}{l}\text { Henderson } \\
\text { and Pabis }\end{array}$ & $M R=a \exp (-k t)$ & $\begin{array}{l}\text { (Henderson and } \\
\text { Pabis, 1961) }\end{array}$ \\
\hline Logarithmic & $\begin{array}{l}M R \\
=a \exp (-k t)+c\end{array}$ & (Toğrul, 2005) \\
\hline Two-term & $\begin{array}{l}M R \\
=a \exp \left(-k_{0} t\right) \\
+b \exp \left(-k_{1} t\right)\end{array}$ & (Henderson, 1974) \\
\hline $\begin{array}{l}\text { Two-term } \\
\text { exponential }\end{array}$ & $\begin{array}{l}M R \\
=a \exp (-k t)+(1 \\
-a) \exp (-k a t)\end{array}$ & $\begin{array}{l}\text { (Y. I. Sharaf- } \\
\text { Eldeen, J. L. } \\
\text { Blaisdell and M. Y. } \\
\text { Hamdy, 1980) }\end{array}$ \\
\hline $\begin{array}{l}\text { Wang and } \\
\text { Singh }\end{array}$ & $M R=1+a t+b t^{2}$ & $\begin{array}{l}\text { (Wang and Singh, } \\
1978 \text { ) }\end{array}$ \\
\hline Weibull & $\begin{array}{l}M R \\
=\exp (-t / \beta)^{\alpha}\end{array}$ & $\begin{array}{l}\text { (Tzempelikos et al., } \\
\text { 2015) }\end{array}$ \\
\hline
\end{tabular}

Tabla 1 Modelos matemáticos aplicados al proceso de secado solar

\section{Análisis estadístico}

El coeficiente de determinación $\left(R^{2}\right)$ se utilizó en este estudio como uno de los criterios primarios para seleccionar el modelo más preciso en comparación con los datos experimentales (Midilli, Kucuk and Yapar, 2002). $R^{2}$ se evaluó calculando los diferentes parámetros involucrados en los modelos de ajuste seleccionados utilizando la versión de software DataFit 9,1. Además, la ji-cuadrado reducida $\left(\chi^{2}\right)$ y el error raíz-medio-cuadrado (RMSE) fueron utilizados como criterios aptos para los datos. El RMSE proporcionó la desviación entre los valores experimentales y predichos, con menos desviación indicada por valores más cercanos a cero. El modelo con el coeficiente de determinación más alto y el RMSE más bajo fue seleccionado como el mejor modelo para describir la cinética de sequía de las hojas del orégano. Se puede lograr un mejor ajuste si el valor de $\left(\chi^{2}\right)$ se reduce según la literatura (Sarracino M. et al., 2018).

El análisis estadístico se realizó mediante las siguientes ecuaciones:

$$
\begin{aligned}
& R^{2}=\frac{\sum_{i=1}^{n}\left(M R_{1}-M R_{\text {pre }, i}\right) \sum_{i=1}^{n}\left(M R_{1}-M R_{\text {exp }, i}\right)}{\sqrt{\left[\sum_{i=1}^{n}\left(M R_{1}-M R_{\text {pre }, i}\right)^{2}\right]\left[\sum_{i=1}^{n}\left(M R_{1}-M R_{\text {exp }, i}\right)^{2}\right]}} \\
& R M S E=\sqrt{\frac{1}{N} \sum_{i=1}^{N}\left(M R_{\text {pre }, i}-M R_{\text {exp }, i}\right)^{2}} \\
& \chi^{2}=\frac{\sum_{i=1}^{N}\left(M R_{\text {exp }, i}-M R_{\text {pre }, i}\right)^{2}}{N-n}
\end{aligned}
$$

Donde $M R_{\text {exp,i }}$ denota la relación de humedad experimental, $M R_{\text {pre }, i}$ es la relación de humedad predicha por el modelo matemático, $\mathrm{N}$ es el número de observaciones, y $n$ es el número de constantes.

\section{Resultados y discusión}

Durante el período de prueba, se realizaron aproximadamente 20 experimentos; Sin embargo, en la mayoría de los casos, se observó un cierto nivel de reproducibilidad, especialmente en relación con los valores medios de irradiación solar en los días de prueba. Por lo tanto, varios días de prueba representativos fueron seleccionados del 3 al 7 de agosto, 2019.

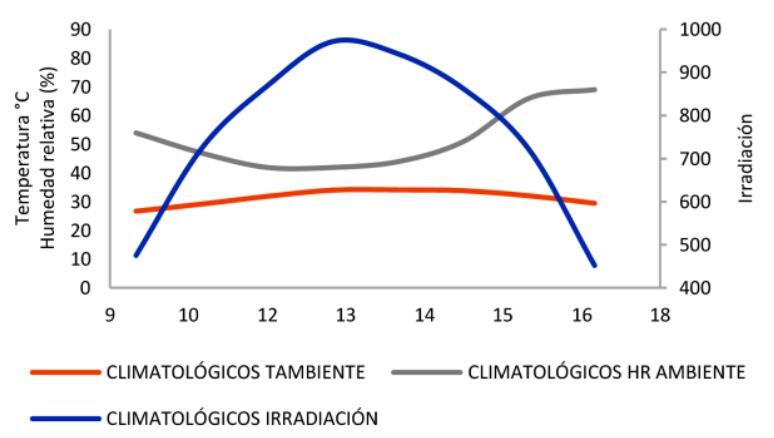

Figura 3 Variación en la irradiancia solar y la temperatura dentro de la cámara de secado bajo tres modos DSD

La figura 3 muestra el cambio en la irradiación solar recibida de aproximadamente $1000 \mathrm{~W} / \mathrm{m}^{2}$. También se midieron las variaciones de temperatura dentro de las cámaras de secado. Con el secado por convección natural, la temperatura interna osciló generalmente entre 40 y $50{ }^{\circ} \mathrm{C}$, con un valor máximo de $60{ }^{\circ} \mathrm{C}$.

En el caso del secado por convección forzada, la temperatura interna generalmente osciló entre 30 y $35^{\circ} \mathrm{C}$, con un valor máximo de $40^{\circ} \mathrm{C}$. Cuando la cámara de secado se cubrió con malla sombreada, las temperaturas medias oscilaron entre 35 y $40{ }^{\circ} \mathrm{C}$, con un valor máximo de $43{ }^{\circ} \mathrm{C}$.

\section{Cinética de secado solar}

Los valores iniciales y finales de humedad y actividad de agua de las hojas frescas y secas se enumeran en la Tabla 2 para cada modo de convección, incluyendo el sol abierto y el secador solar indirecto. 


\begin{tabular}{|l|r|r|r|r|}
\hline Tipo de secado & \multicolumn{2}{c|}{ Humedad (\%) } & \multicolumn{2}{c|}{$\begin{array}{c}\text { Actividad del } \\
\text { agua (aw) }\end{array}$} \\
& \multicolumn{1}{c|}{ Inicial } & \multicolumn{1}{c|}{ Final } & \multicolumn{2}{c|}{$\begin{array}{c}\text { Inicial } \\
\text { Final }\end{array}$} \\
\hline $\begin{array}{l}\text { Convección } \\
\text { natural }\end{array}$ & 80.30 & 8.93 & 0.856 & 0.178 \\
\hline $\begin{array}{l}\text { Convección } \\
\text { forzada }\end{array}$ & 81.25 & 9.01 & 0.875 & 0.198 \\
\hline Cielo abierto & 80.43 & 8.73 & 0.845 & 0.163 \\
\hline $\begin{array}{l}\text { Secador solar } \\
\text { indirecto }\end{array}$ & 81.87 & 9.32 & 0.883 & 0.179 \\
\hline
\end{tabular}

Tabla 2 Humedad inicial y final y actividad del agua obtenida por diferentes métodos de secado (promedio)

Como se puede observar, en todos los casos se detectó poca variación en la humedad inicial. Se informaron niveles de humedad final ligeramente inferiores a los obtenidos por productos comerciales. También se puede observar que el aumento similar en los niveles de humedad se obtuvo en cada prueba independientemente de la operación de secado y/o el método. Se observó una tendencia similar con respecto a las diferencias en la actividad del agua. Los resultados de la actividad final del agua obtenida por todas las tecnologías de secado probadas indican que las condiciones ambientales no imponen efectos nocivos sobre las muestras deshidratadas. La figura 4 muestra el cambio en los parámetros meteorológicos durante el período de prueba con tres días soleados. Como se puede ver, una irradiación global solar máxima de $1000 \mathrm{~W} / \mathrm{m}^{2}$ se alcanzó, con los valores máximos medios que oscilaban entre 950 y $980 \mathrm{~W} / \mathrm{m}^{2}$.

La temperatura ambiente mínima promedio fue de $33{ }^{\circ} \mathrm{C}$, mientras que la temperatura ambiente máxima promedio fue de $3{ }^{\circ} \mathrm{C}$. Por otro lado, el RH mínimo osciló entre 52 y $55 \%$, mientras que el RH máximo osciló entre 32.65 y $50 \%$.

\section{Contenido de humedad (kg agua/kg materia seca)}

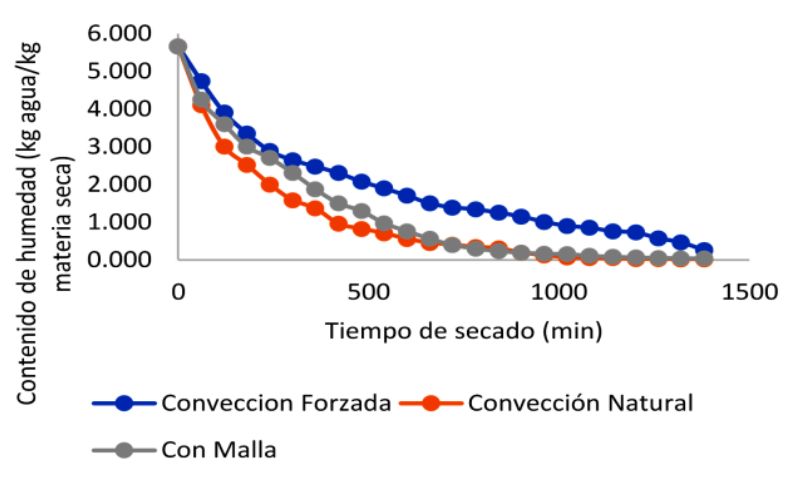

Figura 4 Contenido de humedad con secado solar directo con y sin convección forzada y con sombra malla
La Figura 4 muestra el cambio en el contenido de humedad en d.b. en secadores solares directos que operan con convección natural y forzada, y malla de sombra. Se observó cinética de secado más rápido bajo convección natural, debido al pobre intercambio de calor entre las hojas de orégano y la baja velocidad del aire dentro de la cámara de secado durante este proceso de secado.

La pérdida de peso de las hojas de orégano alcanzó estabilidad después del secado durante 1000 minutos por exposición a la luz solar.

Se alcanzó una temperatura más baja utilizando DSD con convección forzada debido al movimiento de aire saturado, que extrae el calor de la cámara de secado. Así, la cinética de secado se estabilizó a 1380 min en este caso. La curva de secado obtenida mediante malla de sombra reveló una cinética de secado más rápida que la obtenida mediante convección forzada con un tiempo de secado de $1200 \mathrm{~min}$. Este resultado indica que el uso de malla de sombra para obstruir el $60 \%$ de la irradiación solar condujo a un tiempo de secado más largo que el obtenido por convección natural, pero sin utilizar energía eléctrica.

\section{Secado a cielo abierto}

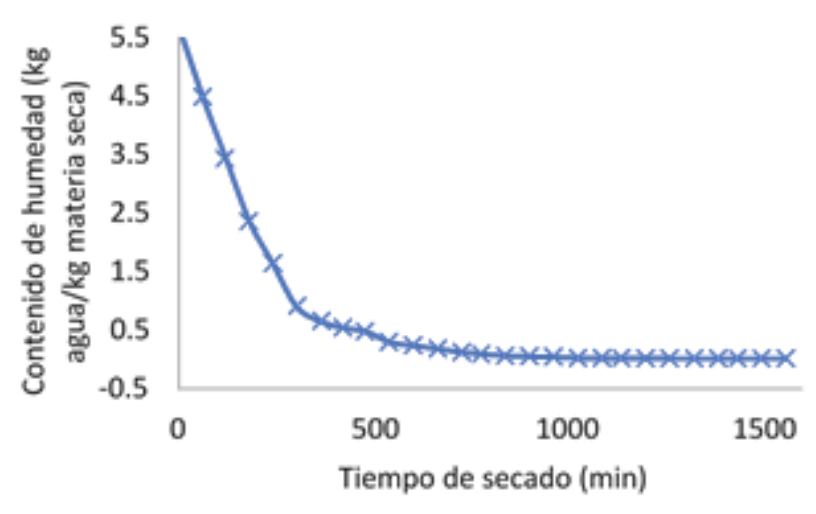

Figura 5 Variación en el contenido de humedad durante el secado a cielo abierto

La Figura 5 muestra la variación en el contenido de humedad durante la prueba de secado solar abierto. La pérdida de peso de la muestra alcanzó un equilibrio entre 2160 y $2340 \mathrm{~min}$, con un peso final de la muestra de $0,801 \mathrm{~g}$ obtenido después del secado durante un promedio de 39,17 horas de sol. 


\section{Secado solar indirecto (ISD)}

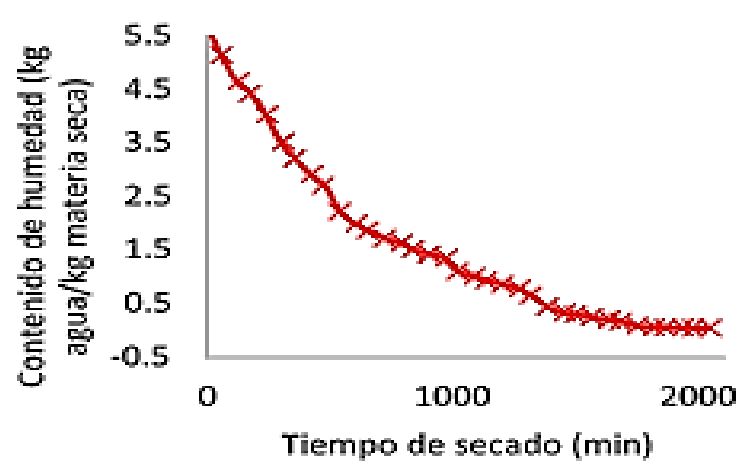

Figura 6 Variación del contenido de humedad en el secador indirecto

La Figura 6 muestra la variación en la irradiación solar en comparación con las temperaturas medidas dentro de la cámara de secado y el colector solar durante el período de prueba del 3 al 7 de agosto de 2019. Como se puede ver, una irradiación solar máxima de $1000 \mathrm{~W} / \mathrm{m}^{2}$. con un valor máximo promedio que oscila entre 800 y $900 \mathrm{~W} / \mathrm{m}^{2}$.

La temperatura máxima alcanzada en la cámara de secado fue de $54{ }^{\circ} \mathrm{C}$ con un promedio de $50{ }^{\circ} \mathrm{C}$. Por otro lado, la temperatura del colector solar fue considerablemente más alta, con un máximo de $65^{\circ} \mathrm{C}$ y una media de $62{ }^{\circ} \mathrm{C}$.

La muestra probada mostró un cambio de peso moderadamente rápido en un corto período de tiempo, ya que el peso de la muestra disminuyó de $20 \mathrm{~g}$ a aproximadamente $6 \mathrm{~g}$ durante el tiempo de secado, es importante mencionar que en promedio la muestra en estudio perdió mucho peso entre 0 minutos y d 400 min logrando la estabilización después de $800 \mathrm{~min}$. Figura 6 muestra el cambio en el contenido de humedad basado en el tiempo de secado cuando se utilizó ISD.

Como se puede ver, el contenido de humedad disminuyó significativamente de 5.66 a $1.64 \mathrm{~kg}$ de agua/kg de materia seca, debido a la irradiancia solar recibida por la cámara de secado. A continuación, se observó una disminución lenta y muy homogénea del contenido de humedad, que puede atribuirse a la uniformidad del aire caliente que entra en la cámara de secado desde el colector solar durante las horas pico de insolación. El contenido de humedad de equilibrio se alcanzó en $1300 \mathrm{~min}$.
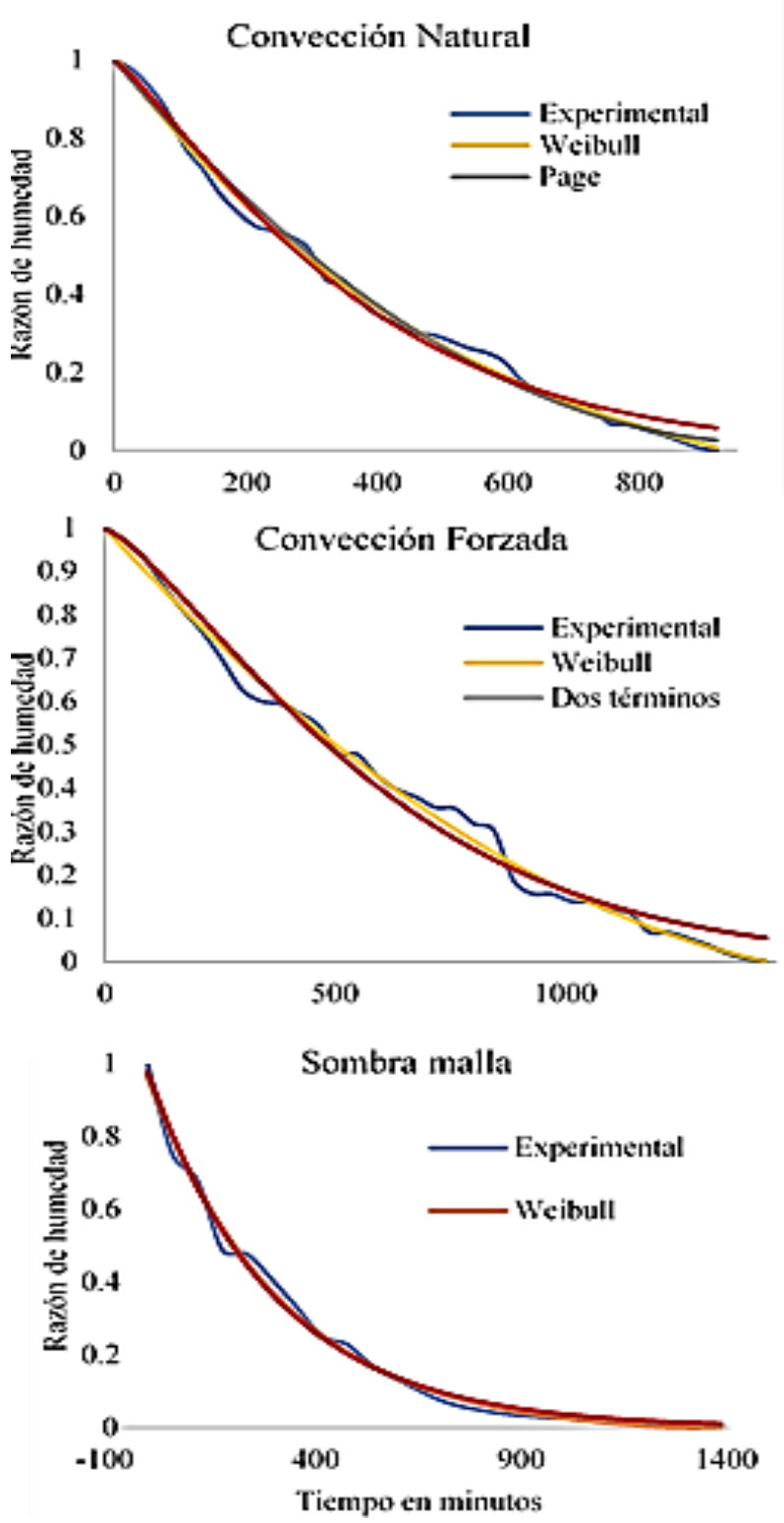

Figura 7 Ajuste de la relación de humedad experimental versus el tiempo de secado obtenido para el DSD bajo A) convección natural, B) convección forzada, C) malla sombreada, con diferentes modelos

Los resultados experimentales mostraron que el uso de secador solar directo con convección natural reduce significativamente los tiempos de secado. Cuando el aire se calienta, su densidad disminuye, lo que lleva a que extrae humedad del producto para secar en menos tiempo. Sin embargo, se observó la existencia de pigmentación marrón desigual que aparecen antes en las hojas. Por lo tanto, la calidad del producto final disminuye. En el caso de la convección forzada, donde, el aire húmedo se extrae más rápido de la cámara de secado, aumentando el tiempo de secado. Sin embargo, se observó visualmente que las hojas conservan menos degradación del color. 
El secado de sol abierto necesitaba prácticamente duplicar el tiempo en comparación con el resto de las tecnologías, además de todos los inconvenientes relacionados con la higiene del producto. Finalmente, mediante la adición de un colector solar fue posible transmitir calor al ISD y disminuir el tiempo de secado debido al enredo de aire caliente dentro de la cámara de secado, también se observó visualmente que la coloración de las muestras se conservaba, esto de acuerdo con resultados, con mayor será la temperatura de secado, menor será la estabilidad de los pigmentos.

\section{Modelado matemático del proceso de secado}

En este estudio se analizaron nueve modelos predictivos, como se indica en la Tabla 1. Las constantes correspondientes y los parámetros de ajuste $r^{2}$ y $\chi^{2}$ se obtuvieron valores para cada modelo. Los resultados experimentales de la curva de tiempo de secado y el MR son comparados mediante análisis de regresión. Los valores más cercanos a 1 para $\mathrm{r}^{2}$ de los modelos explorados, así como los menores valores para RMSE y $\chi^{2}$ son los que representan de mejor manera el comportamiento del secado solar de hojas de orégano.

\begin{tabular}{|c|c|c|}
\hline Modelo & $\begin{array}{c}\text { Coeficientes y } \\
\text { parámetros de ajuste }\end{array}$ & Valor \\
\hline \multicolumn{3}{|c|}{ Convección natural } \\
\hline \multirow[t]{8}{*}{ Weibull } & $a$ & 0.0036 \\
\hline & $b$ & - \\
\hline & $k$ & 0.9986 \\
\hline & $n$ & 0.5193 \\
\hline & & 1.2143 \\
\hline & $r^{2}$ & 0.9982 \\
\hline & $R M S E$ & 0.0114 \\
\hline & $X^{2}$ & 0.0155 \\
\hline \multirow[t]{5}{*}{ Page modificado } & $k$ & 0.5777 \\
\hline & $n$ & 1.2022 \\
\hline & $r^{2}$ & 0.9981 \\
\hline & $R M S E$ & 0.0118 \\
\hline & $X^{2}$ & 6.3689 \\
\hline \multirow[t]{5}{*}{ Page } & $k$ & 0.5170 \\
\hline & $n$ & 1.2022 \\
\hline & $r^{2}$ & 0,9981 \\
\hline & $R M S E$ & 0.3864 \\
\hline & $X^{2}$ & 6.3689 \\
\hline \multicolumn{3}{|c|}{ Convección forzada } \\
\hline \multirow[t]{7}{*}{ Dos términos } & $a$ & 0.2674 \\
\hline & $b$ & 0.7314 \\
\hline & $c$ & 1.0980 \\
\hline & $d$ & 0.0846 \\
\hline & $r^{2}$ & 0.9895 \\
\hline & $R M S E$ & 0.0231 \\
\hline & $X^{2}$ & 0.0690 \\
\hline
\end{tabular}

\begin{tabular}{|c|c|c|}
\hline \multirow[t]{7}{*}{ Weibull } & $a$ & 1.3824 \\
\hline & $b$ & 2.3855 \\
\hline & $k$ & 0.1183 \\
\hline & $n$ & 0.4532 \\
\hline & $r^{2}$ & 0.9922 \\
\hline & $R M S E$ & 0.0199 \\
\hline & $X^{2}$ & 0.0406 \\
\hline \multirow{5}{*}{ Page } & $k$ & 0.2477 \\
\hline & $n$ & 0.6854 \\
\hline & $r^{2}$ & 0.9839 \\
\hline & $R M S E$ & 0.0286 \\
\hline & $X^{2}$ & 0.1022 \\
\hline \multicolumn{3}{|l|}{ Sombra-malla } \\
\hline \multirow[t]{7}{*}{ Weibull } & $a$ & 0.0295 \\
\hline & $b$ & 1.0114 \\
\hline & $K$ & 0.2039 \\
\hline & $n$ & 0.9396 \\
\hline & $r^{2}$ & 0.9937 \\
\hline & $R M S E$ & 0.0215 \\
\hline & $X^{2}$ & 0.1163 \\
\hline \multirow{7}{*}{ Logarítmico } & $a$ & 0.9856 \\
\hline & $c$ & \\
\hline & $k$ & 0.0180 \\
\hline & & 0.1845 \\
\hline & $r^{2}$ & 0.9997 \\
\hline & RMSE & 0.0220 \\
\hline & $X^{2}$ & $\begin{array}{l} \\
0.0345\end{array}$ \\
\hline \multirow{5}{*}{$\begin{array}{l}\text { Henderson and } \\
\text { Pabis }\end{array}$} & $a$ & 0.9770 \\
\hline & $k$ & 0.1959 \\
\hline & $r^{2}$ & 0.9924 \\
\hline & $R M S E$ & 0.0235 \\
\hline & $X^{2}$ & 0.0747 \\
\hline
\end{tabular}

Tabla 3 Coeficientes y parámetros de ajuste para DSD (convección natural y forzada y sombra-malla)

Las tablas 3, 4 y 5 presentan los coeficientes y parámetros de ajuste de los resultados experimentales obtenidos utilizando los modelos matemáticos para analizar el DSD, el sol abierto y la cinética de secado ISD, respectivamente, con el fin de obtener los mejores ajustes.

\begin{tabular}{|l|l|l|}
\hline Modelo & $\begin{array}{l}\text { Coeficientes y parámetros } \\
\text { de ajuste }\end{array}$ & Valor \\
\hline Weibull & $a$ & 0.0076 \\
\cline { 2 - 3 } & $b$ & 0.9942 \\
& $k$ & 0.2310 \\
& $n$ & 1.2287 \\
\cline { 2 - 3 } & $r^{2}$ & 0,9981 \\
\cline { 2 - 3 } & $R M S E$ & 0.0111 \\
\cline { 2 - 3 } & $X^{2}$ & 0.0747 \\
\hline Page Modificado & $k$ & 0.2982 \\
& $n$ & 1.2022 \\
\cline { 2 - 3 } & $r^{2}$ & 0,9976 \\
\cline { 2 - 3 } & $R M S E$ & 0.0125 \\
\cline { 2 - 3 } & $X^{2}$ & 1.8242 \\
\hline Page & $k$ & 0.2339 \\
& $n$ & 1.2006 \\
\cline { 2 - 3 } & $r^{2}$ & 0,9976 \\
\cline { 2 - 3 } & $R M S E$ & 0.0125 \\
\cline { 2 - 3 } & $X^{2}$ & 1.8242 \\
\hline
\end{tabular}

Tabla 4 Coeficientes y parámetros de ajuste para el secado cielo abierto

CASTILLO-TÉLLEZ, Beatriz, CASTILLO-TÉLLEZ-Margarita, MEJÍA-PÉREZ, Gerardo Alberto y LÓPEZ VIDAÑA, Erick César. Modelado matemático de secado solar de orégano (Plectranthus amboinicus) con tecnologías directas e indirectas. Revista de Tecnologías en Procesos Industriales. 2020 
El mejor ajuste para el DSD con la convección natural e logró mediante Weibull, Page y Page modificado, con $\mathrm{R}^{2}$ de 0.9982 , 0.9981 y 0.9981 ; y un $X^{2}$ de $0.0155,6.3689$ y 0.0406 respectivamente. Mientras tanto, los mejores resultados con la convección forzada fueron obtenidos por Dos términos, Weibull, y Page, con $\mathrm{R}^{2}$ de $0.9895,0.9922$ y 0.9839 ; $\mathrm{Y} X^{2}$ de $0.0690,0.0406$, y 0.1022, respectivamente. El mejor ajuste para DSD con malla de sombra fue obtenido por Weibull, logarítmico y Henderson, y modelos Pabis, con $\mathrm{R}^{2}$ de $0.9937,0.9997$ y 0.9924; y un $X^{2}$, de $0.1163,0.0345$, y 0.0747 , respectivamente.

\begin{tabular}{|l|l|r|}
\hline \multicolumn{1}{|c|}{ Modelo } & \multicolumn{1}{c|}{$\begin{array}{c}\text { Coeficientes y } \\
\text { parámetros de ajuste }\end{array}$} & \multicolumn{1}{c|}{ Valor } \\
\hline \multirow{5}{*}{ Weibull } & $a$ & -0.0737 \\
\cline { 2 - 3 } & $b$ & -1.0801 \\
& $k$ & 0.0932 \\
& $n$ & 0.9612 \\
\cline { 2 - 3 } & $r^{2}$ & 0,9969 \\
\cline { 2 - 3 } & $R M S E$ & 0.0150 \\
\cline { 2 - 3 } & $X^{2}$ & 0.0363 \\
\hline \multirow{5}{*}{ logarítmico } & $a$ & 1.0554 \\
& $c$ & -0.0585 \\
& $k$ & 0.0863 \\
\cline { 2 - 3 } & $r^{2}$ & 0,9968 \\
\cline { 2 - 3 } & $R M S E$ & 0.0153 \\
\cline { 2 - 3 } & $X^{2}$ & 0.0392 \\
\hline \multirow{5}{*}{ Page } & $k$ & 0.0800 \\
& $n$ & 1.0851 \\
\cline { 2 - 3 } & $r^{2}$ & 0,9945 \\
\cline { 2 - 3 } & $R M S E$ & 0.0170 \\
\cline { 2 - 3 } & $X^{2}$ & 0.0599 \\
\hline
\end{tabular}

Tabla 5 Coeficientes y parámetros del ajuste para el ISD

El modelo que representa de mejor manera el comportamiento para el secado a cielo abierto fue Weibull, Page modificado, y Page, con $\mathrm{R}^{2}$ de $0.9981,0.9976$ y 0.9976 ; $\mathrm{Y} X^{2}$ de $0.0747,1.8242$, y 1.8242 , respectivamente. Mientras tanto, el mejor ajuste para el ISD fue obtenido por los modelos Weibull, logarítmicos, y de la Page, con $R^{2}$ de $0.9969,0.9968$ y 0.9945 ; $Y X^{2}$ de $0.0363,0.0363$ y 0.0599 .

La validación del modelo establecido se realiza comparando la relación de humedad pronosticada con la relación de humedad experimental.

Las figuras 7 y 8 muestran el ajuste de los datos experimentales de secado solar de hojas de orégano utilizando las tecnologías de secado solar directa e indirecta probadas con diferentes modelos matemáticos.

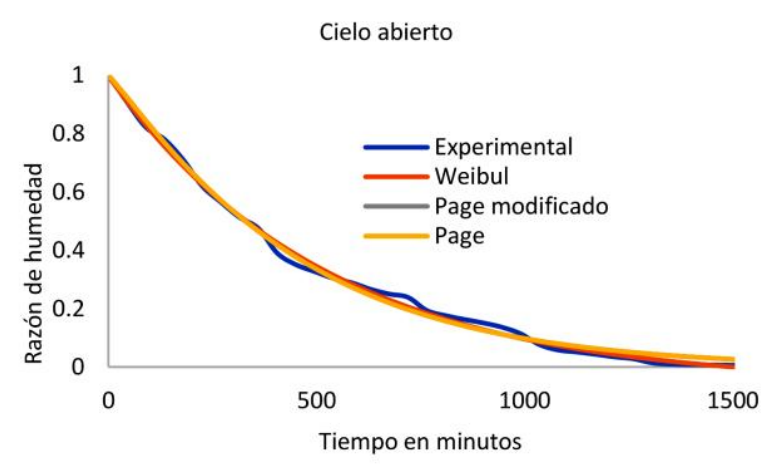

Figura 8 Ajuste de la relación de humedad experimental con modelos matemáticos

\section{Conclusiones}

En este estudio, la cinética de secado de las hojas de orégano se analizó utilizando las tecnologías DSD y ISD. Los resultados revelaron una calidad superior de los productos secos obtenidos usando ISD, con tiempos de secado entre 1100 y 1200 minutos para alcanzar un contenido de humedad de la materia seca de 0.013 kilogramos agua $/ \mathrm{kg}$ de materia seca y una tasa de secado de 0.001 kilogramos de materia seca del agua/kilogramo min, con un promedio tiempo de secado entre el que se obtiene utilizando DSD bajo modos de cobertura de convección natural y malla sombreada. Además, las condiciones de funcionamiento fueron mejor controladas y se mantuvo una mayor protección contra los efectos de la temperatura debido a la exposición indirecta a la radiación solar.

Un colector solar plano naturalmente funcionado fabricado con los materiales económicos (madera negra y vidrio 3 milímetros de grueso) fue acoplado con el ISD para alcanzar calidad de producto secado aceptable con costo mínimo. Por lo tanto, el ISD se puede considerar como método de secado simple y económico. Aunque el uso de convección natural redujo el tiempo de secado con temperaturas de operación más altas, se detectó pigmentación marrón, que afecta negativamente la calidad del producto seco. La cubierta de malla negra que se integró mejoró la calidad de las hojas secas, redujo el efecto de la descoloración, y disminuyó los tiempos de sequía comparados a los obtenidos por la convección forzada. Se probaron nueve modelos matemáticos para determinar los parámetros de los modelos apropiados para predecir el comportamiento de secado de las plantas de orégano. 
Los modelos Weibull, Page y logarítmicos fueron los más aptos para los datos experimentales obtenidos utilizando ISD, con $\mathrm{R}^{2}$ de $0.9969,0.9968$, y 0.9945 , y $X^{2}$ de 0.0363 , 0,0363 y 0.0599 , respectivamente; así, el contenido de humedad en cualquier momento durante el proceso de secado podría ser estimado confiablemente usando estos modelos. Los hallazgos confirman la viabilidad técnica de utilizar tecnologías de secado solar para hojas de orégano. Se pueden lograr ahorros energéticos significativos evitando el uso de energía convencional durante el proceso de secado, minimizando así los impactos ambientales.

\section{Referencias}

Castillo Téllez, M. et al. (2018) 'Solar drying of Stevia (Rebaudiana Bertoni) leaves using direct and indirect technologies', Solar Energy. Elsevier, 159(November 2017), pp. 898-907. doi: 10.1016/j.solener.2017.11.031.

Chouicha, S. et al. (2013) 'Solar drying of sliced potatoes.an experimental investigation', Energy Procedia. Elsevier B.V., 36, pp. 1276-1285. doi: 10.1016/j.egypro.2013.07.144.

Doymaz, I. and Smail, O. (2011) 'Drying characteristics of sweet cherry', Food and Bioproducts Processing, 89(1), pp. 31-38. doi: 10.1016/j.fbp.2010.03.006.

Fudholi, A. et al. (2014) 'Performance analysis of solar drying system for red chili', Solar Energy. Elsevier Ltd, 99, pp. 47-54. doi: 10.1016/j.solener.2013.10.019.

Henderson, S. M. (1974) 'PROGRESS IN DEVELOPING THE THIN LAYER DRYING EQUATION.', Transactions of the American Society of Agricultural Engineers, 17(6).

Henderson, S. M. and Pabis, S. (1961) 'Grain drying theory II: Temperature effects on drying coefficients', Journal of Agricultural Engineering Research, 6(3), pp. 169-174.

Hossain, M. B. et al. (2010) 'Effect of drying method on the antioxidant capacity of six Lamiaceae herbs', Food Chemistry. Elsevier Ltd, 123(1), pp. 85-91. doi: 10.1016/j.foodchem.2010.04.003.
Liu, Q. and Bakker-Arkema, F. W. (1997) 'Stochastic modelling of grain drying: Part 2. Model development', Journal of Agricultural and Engineering Research, 66(4), pp. 275-280. doi: 10.1006/jaer.1996.0145.

Méndez-Robles, L. I. et al. (2018) Análisis de propiedades físicas en el deshidratado de Guayaba por medio de energía solar utilizando convección natural y forzada. RESUMEN. Available

at: http://www.fcb.uanl.mx/IDCyTA/files/volume3 14/3/45.pdf (Accessed: 27 August 2019).

Midilli, A., Kucuk, H. and Yapar, Z. (2002) 'A new model for single-layer drying', Drying Technology, 20(7), pp. 1503-1513. doi: 10.1081/DRT-120005864.

Mueller, M. et al. (2008) 'Oregano: a source for peroxisome proliferator-activated receptor gamma antagonists.', Journal of agricultural and food chemistry, 56, pp. 11621-11630. doi: 10.1021/jf802298w.

Ouaabou, R. et al. (2018) 'Valorization of solar drying process in the production of dried Moroccan sweet cherries', Solar Energy, 172(November 2017), pp. 158-164. doi: 10.1016/j.solener.2018.05.079.

Page, G. E. (1949) Factors influencing the maximum rates of air drying shelled corn in thin layers. M. S. Thesis. Purdue University, West Lafayette, IN, USA.

Sarracino M., O. et al. (2018) 'Experimental study of dried Oregano leaves (Plectranthus amboinicus, Lour) using direct and indirect solar technologies', Drying Technology Experimental, 33(3), pp. 321-332. doi: $10.1080 / 1478643 \mathrm{YY}$.

Tlatelpa-Becerro, A. et al. (2018) 'TGuava thin layer drying kinetics for an indirect solar dryer', in XXIV CONGRESO INTERNACIONAL ANUAL DE LA SOMIM. Campeche, Campeche, pp. 163-168.

Toğrul, H. (2005) 'Simple modeling of infrared drying of fresh apple slices', Journal of Food Engineering, 71(3), pp. 311-323. doi: 10.1016/j.jfoodeng.2005.03.031. 
Tunde-Akintunde, T. Y. (2011) 'Mathematical modeling of sun and solar drying of chilli pepper', Renewable Energy. Elsevier Ltd, 36(8), pp. 2139-2145. doi: 10.1016/j.renene.2011.01.017.

Tzempelikos, D. A. et al. (2015) 'Experimental study on convective drying of quince slices and evaluation of thin-layer drying models', Engineering in Agriculture, Environment and Food. Elsevier Ltd, 8(3), pp. 169-177. doi: 10.1016/j.eaef.2014.12.002.

Vázquez, F. (2016) Óregano, el oro verde del norte de Jalisco - Jaliscocina, Periodismo Cultural Gastronómico. Available at: https://jaliscocina.com/oregano-el-oro-verdedel-norte-de-jalisco (Accessed: 30 August 2018).

Vázquez Martínez, A. (2010) Orégano: oro verde de México - Info Rural. Available at: https://www.inforural.com.mx/oregano-oroverde-de-mexico/ (Accessed: 6 July 2018).

Wang, C. Y. and Singh, R. P. (1978) 'A single layer drying equation for rough rice', American Society of Agricultural Engineers, (78), p. 3001. doi: 10.1081/E-EEE2-120046011.

White, G. M., Ross, I. J. and Poneleit, C. G. (1981) 'Fully-exposed drying of popcorn', European Physical Education Review, 24(2), pp. 466-468, $475 . \quad$ doi: http://dx.doi.org/10.1108/17506200710779521.

Y. I. Sharaf-Eldeen, J. L. Blaisdell and M. Y. Hamdy (1980) 'A Model for Ear Corn Drying', Transactions of the ASAE, 23(5), pp. 12611265. doi: 10.13031/2013.34757. 